\title{
Asymptomatic carriage of Clostridium difficile PCR ribotype 078 in pigs
}

\author{
L A Brunton, J S Knapp, J Heritage, H M Miller
}

University of Leeds, Leeds, United Kingdom

Email:bmb3lab@leeds.ac.uk

Introduction Clostridium difficile has been reported to be the most common cause of neonatal enteritis in pigs in the United States (Songer and Anderson 2006) and there are a number of reports of its presence in pigs in Europe (Nagy and Bilkei 2003, Songer and Anderson 2006, Debast et al. 2009). In contrast, it has been observed in previous experimental work at the University of Leeds pig farm that the incidence of asymptomatic $C$. difficile carriage in pigs is common in young pigs but rare in pigs at slaughter age (Brunton et al., 2009). The aim of this study was to observe the incidence of $C$. difficile carriage in piglets at weaning through to 18 weeks of age. We hypothesised that asymptomatic $C$. difficile carriage would be high in pigs at weaning, but would decrease with age. Our second hypothesis was that the addition of a therapeutic level of an antibiotic, phenoxymethyl penicillin, to the pigs diet would increase the incidence of $C$. difficile carriage. Antibiotic treatment is a risk factor for the onset of $C$. difficile associated infection (CDI) in humans (Bignardi 1998).

Materials and methods Thirty-six piglets from six litters were distributed across the two treatments. Pigs were allocated across six pens (six pigs in each) so that pens were balanced for litter, gender and weight (average weight $=7.7 \mathrm{~kg} \pm 0.93$ SD). All pigs were fed on a standard commercial weaner diet from 4 to 7 weeks of age, after which a standard grower diet was introduced until week 12 . This grower diet contained therapeutic levels of phenoxymethyl penicillin $(0.2 \mathrm{~kg} / \mathrm{tonne}$, prescribed to combat streptococcus suis infections) for pigs in 3 of the pens, whilst the other 3 pens were fed the base diet with no antibiotics. From week 12 onwards all pigs were fed a standard commercial finisher diet containing no antibiotics. Faecal samples were collected from all 36 pigs at 4 weeks (weaning), and then at 7, 10, 12, 13 and 18 weeks of age. The health of the animals was monitored throughout the study. DNA was extracted from each of the faecal samples using a phenol:chloroform based method. C. difficile DNA was detected using the polymerase chain reaction (PCR) with probes specific for the $t c d A$ gene which encodes the toxin A. PCR results were analysed using the chi squared test including Yates' correction for two categories of data. PCR positive samples were cultured in an attempt to isolate $C$. difficile. Ribotyping of the isolates was carried out by the Clostridium difficile Ribotyping Network (CDRN) based at the Leeds General Infirmary, Leeds, West Yorkshire. A cytotoxin assay was also carried out on all samples in which the $t c d A$ gene had been detected using PCR.

Results At week 4, all of the treatment group (18/18) and 16/18 of the control group screened positive for C. difficile. All animals were asymptomatic and there was no statistically significant difference between the two groups. By week 7, the number of pigs that were positive for $C$. difficile had decreased significantly for both groups to only 2 positive samples for the treatment group and 3 positive samples for the control group ( $\mathrm{P}<0.001$ and $\mathrm{P}<0.01$ respectively) (Figure 1$)$. The number of positive samples remained low for the rest of the trial. There was no significant difference between groups across the trial. Of the 46 samples that were screened as positive by PCR, 13 yielded C. difficile when cultured. Eight of the 13 were from pigs in the treatment group, and the remaining 5 were from pigs in the control group. All 13 isolates were ribotyped and found to be the PCR ribotype 078. None of the samples demonstrated significant cytotoxic effects.

Conclusions We have demonstrated that on the UK farm studied asymptomatic carriage of $C$. difficile is common, but short-lived in piglets. The inclusion of phenoxymethyl penicillin in the pigs' diet was found to have no effect on the carriage of $C$. difficile. The observations of this study are contradictory to reports from North America and parts of Europe, where $C$. difficile is claimed to cause symptomatic illness in young pigs (Songer and Anderson 2006, Debast et al. 2009). The phenomenon of asymptomatic carriage which decreases with age has been observed in other species, including humans and rabbits, but has not before been demonstrated in pigs. Further work is required to determine the reasons for this phenomenon, and to understand why $C$. difficile is a problem in North America, but not in the UK.

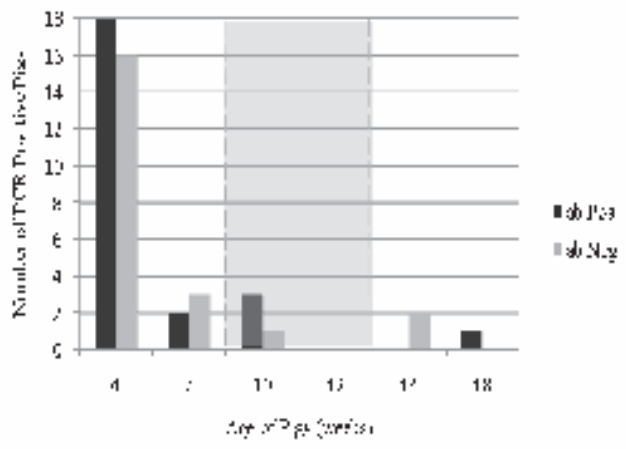

Acknowledgements The authors wish to thank Professor Mark Wilcox and his group for their assistance with the culture and ribotyping of isolates, and cytotoxicity assays. Lucy Brunton was supported by a BPEX PhD studentship.

\section{References}

Bignardi G. E, 1998. Journal of Hospital Infections 40, 1.

Brunton L., J. Knapp, J. Heritage, H.M. Miller, 2009. Proceedings of the British Society of Animal Science.

Debast, S., L. van Leengoed, A. Goorhuis, C. Harmanus, E. Kuijper, A. Bergwerff, 2009. Environmental Microbiology 11, 505.

Nagy and Bilkei, 2003. Vet. Journal 166, 98.

Songer and Anderson, 2006. Anaerobe. 12, 1. 